
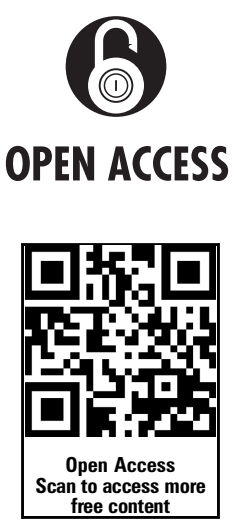

Handling editor Tore K Kvien

- Additional material is published online only. To view please visit the journal online (http://dx.doi.org/10.1136/ annrheumdis-2013-203936)

${ }^{1}$ University of Massachusetts Medical School, Worcester,

Massachusetts, USA ${ }^{2}$ Albany Medical College, Albany, New York, USA ${ }^{3}$ Department of Immunology and Rheumatology, University of Alabama at Birmingham, Birmingham, Alabama, USA ${ }^{4}$ Department of Medicine, Brigham and Women's Hospital, Boston, Massachusetts, USA

${ }^{5}$ Departments of Medicine and Epidemiology and Public Health, University of Maryland School of Medicine, Baltimore, Maryland, USA

${ }^{6}$ Department of Rheumatology, New York University Hospital for Joint Diseases, New York, USA

\section{Correspondence to}

Dr Leslie R Harrold,

Department of Orthopedics,

University of Massachusetts

Medical School, 55 Lake

Ave North, Worcester,

MA 01655, USA;

Leslie.Harrold@umassmed.edu

Received 12 May 2013

Revised 10 September 2013

Accepted 10 November 2013

Published Online First

2 December 2013

\section{CrossMark}

To cite: Harrold $L R$,

Reed GW, Kremer JM, et al. Ann Rheum Dis

2015;74:430-436

\title{
The comparative effectiveness of abatacept versus anti-tumour necrosis factor switching for rheumatoid arthritis patients previously treated with an anti-tumour necrosis factor
}

\author{
Leslie R Harrold, ${ }^{1}$ George W Reed, ${ }^{1}$ Joel M Kremer, ${ }^{2}$ Jeffrey R Curtis, ${ }^{3}$ \\ Daniel H Solomon, ${ }^{4}$ Marc C Hochberg, ${ }^{5}$ Jeffrey D Greenberg ${ }^{6}$
}

\section{ABSTRACT}

Objective We compared the effectiveness of abatacept (ABA) versus a subsequent anti-tumour necrosis factor inhibitor (anti-TNF) in rheumatoid arthritis (RA) patients with prior anti-TNF use.

Methods We identified RA patients from a large observational US cohort (2/1/2000-8/7/2011) who had discontinued at least one anti-TNF and initiated either ABA or a subsequent anti-TNF. Using propensity score (PS) matching (n:1 match), effectiveness was measured at 6 and 12 months after initiation based on mean change in Clinical Disease Activity Index (CDAl), modified American College of Rheumatology (mACR) 20, 50 and 70 responses, modified Health Assessment Questionnaire (mHAQ) and CDAl remission in adjusted regression models.

Results The PS-matched groups included $431 \mathrm{ABA}$ and 746 anti-TNF users at 6 months and 311 ABA and 493 anti-TNF users at 12 months. In adjusted analyses comparing response following treatment with $\mathrm{ABA}$ and anti-TNF, the difference in weighted mean change in CDAl (range $6-8)$ at 6 months $(0.46,95 \% \mathrm{Cl}-0.82$ to $1.73)$ and 12 months was similar $(-1.64,95 \% \mathrm{Cl}$ -3.47 to 0.19$)$. The mACR20 responses were similar at $6(28-32 \%, p=0.73)$ and 12 months (35-37\%, $p=0.48)$ as were the mACR50 and mACR70 (12 months: $20-22 \%, p=0.25$ and $10-12 \%, p=0.49$, respectively).

Meaningful change in $\mathrm{mHAQ}$ was similar at 6 and 12 months (30-33\%, $p=0.41$ and $29-30 \%, p=0.39$, respectively) as was CDAl remission rates $(9-10 \%$, $p=0.42$ and $12-13 \%, p=0.91$, respectively).

Conclusions RA patients with prior anti-TNF exposures had similar outcomes if they switched to a new anti-TNF as compared with initiation of ABA.

\section{INTRODUCTION}

Rheumatoid arthritis (RA) affects an estimated 1.3 million Americans and is associated with substantial morbidity and mortality. ${ }^{1}$ To prevent the development of permanent joint damage and deformity as well as functional impairment, the current treatment paradigm is to treat patients with active disease with disease modifying antirheumatic drugs (DMARDs). ${ }^{2}$ The typical approach is to introduce a conventional non-biologic DMARD first, followed by combination non-biologic DMARDs or biologics in non-responders. ${ }^{3}$ The initial biologic used in the vast majority of patients is the anti-tumour necrosis factor (anti-TNF). These agents were the first class of biologics approved by the Food and Drug Administration (FDA) for the treatment of RA.

However, little is known regarding what to do when patients have an inadequate response to the first anti-TNF agent. Changing mechanism of action has been shown to be beneficial in comparisons of rituximab versus a subsequent anti-TNF ${ }^{4-7}$ as demonstrated by improvements in disease activity as well as persistence of therapy. But it is unclear whether any change in mechanism of action will result in improvement or if the results seen were related specifically to B cell depletion. Further exploration is needed to assess whether switching to abatacept (ABA), as a different example of changing mechanism of action, also provides a benefit among those with inadequate response to an anti-TNF. To date, there have been no randomised controlled trials (RCTs) comparing the effectiveness of switching to ABA as compared with a subsequent anti-TNF, and a recent meta-analysis using indirect methods suggested that use of an anti-TNF was associated with a higher probably of achieving an American College of Rheumatology (ACR) 50 response than ABA, although not all indirect comparisons have shown a difference between ABA and anti-TNF agents. ${ }^{8-10}$ In biologic-naïve patients, a few studies have compared responses to ABA versus an anti-TNF. ${ }^{11-14} \mathrm{~A}$ similar efficacy was found when comparing responses to $\mathrm{ABA}$ and infliximab, although there have been concerns that the results may not be generalisable to the US population of RA patients due to the lower doses of infliximab used $(3 \mathrm{mg} / \mathrm{kg}$ every 8 weeks) and lack of the usual infliximab loading dose. Additionally, ABA (in combination with methotrexate (MTX)) has been compared with adalimumab with MTX among biologic-naïve RA patients with similar ACR response rates. ${ }^{14}$

Given the absence of head-to-head RCTs addressing best treatment approaches in patients with inadequate response to an anti-TNF agent, comparative effectiveness studies using observational data from registries can be employed. ${ }^{15}$ Therefore, the aim of the present study was to compare the clinical effectiveness of ABA versus a subsequent anti-TNF agent among RA patients with previous anti-TNF exposure in a large US cohort of RA patients using the 
Consortium of Rheumatology Researchers of North America (CORRONA) registry. Specifically, we sought to compare change in disease activity, composite response rates, meaningful improvement in function and remission outcomes over a 1-year period.

\section{METHODS}

\section{Data sources and data collection}

CORRONA is a US observational cohort of patients with arthritis who are enrolled by participating rheumatologists in 132 practices both academic and private practice with prospective data collection; the details have been previously described. ${ }^{16} 17$ Data are collected from both patients and their treating rheumatologists, who gather information on disease duration, prognosis, disease severity and activity, medical comorbidities, use of medications including DMARDs and adverse events. ${ }^{18}$ Laboratory data such as inflammatory markers are not available at all visits as they are not mandated due to the observational nature of the registry and some rheumatologists do not obtain this information routinely. Follow-up assessments are requested at least as often as every 4 months and completed during routine clinical encounters. Approvals for participation in the CORRONA registry are obtained from respective Institutional Review Boards (IRBs) of participating academic sites and a central IRB for private practice sites, and patients provide informed consent before enrolment.

\section{Study population}

There were 27412 patients with a diagnosis of RA in the registry. We identified patients with exposure to one or more anti-TNF agents but no prior use of non-anti-TNF biologics using CORRONA data between $2 / 1 / 2000$ and 8/1/2011. For the main analyses, we included all patients regardless of reason for the switch to ABA or a subsequent anti-TNF following prior exposure to an anti-TNF. However, in the sensitivity analyses, we included those patients who were inadequate responders to the prior anti-TNF. Inclusion criteria into the study cohort included receipt of either a subsequent anti-TNF or ABA, a follow-up appointment at 6 and/or 12 months following initiation and the presence of all the elements necessary to calculate response based on the Clinical Disease Activity Index (CDAI), a well-recognised instrument to measure disease activity, and ACR20, 50 and 70 responses. ${ }^{19-21}$ For the purpose of this study, RA patients in remission at baseline, meaning at the time the new agent was initiated, defined by CDAI $\leq 2.8$, were excluded from the study population (switching while in remission is seen when cost or tolerability influences medication use).

\section{Measures and data collection}

Data were collected during the study period from physician assessment and patient questionnaires completed during clinical encounters. Non-biologic and biologic DMARDs, including anti-TNF agents, are recorded at the time of the clinical encounter. Among the data elements collected in the registry relevant to this study are a 28 tender and swollen joint count, physician's and patient's global assessments of disease activity, patient's assessment of pain, the modified Health Assessment Questionnaire (mHAQ) assessing physical function and erythrocyte sedimentation rate (ESR). When available, acute phase reactant data are recorded from laboratory tests obtained within 10 days of the clinical encounter.

\section{Study outcomes}

Responsiveness to medication treatment was defined based on improvement in CDAI as our primary outcome (eg, baseline
CDAI-follow-up CDAI). As a secondary outcome, we evaluated modified ACR20 (mACR20), modified ACR50 (mACR50) and modified ACR70 (mACR70) responses, which do not include acute phase reactants. These measures have been previously defined and validated and shown to highly correlate with ACR responses that include acute phase reactants. ${ }^{20} 22$ An mACR20 response required $\geq 20 \%$ improvement in tender joint count and swollen joint count, as well as two or more of the four remaining ACR response components, including physician's global assessment, patient's global assessment, patient's global pain and mHAQ. The mACR50 and mACR70 responses were calculated using the same criteria requiring $50 \%$ and $70 \%$ improvement, respectively. In addition, we evaluated achievement of a clinically meaningful change in the mHAQ $(\geq 0.25) .^{23}$ Disease remission outcomes were defined using CDAI.

As sensitivity analyses, we examined improvement in the modified Disease Activity Score 28 (mDAS28), which is calculated without results of acute phase reactants as well as achievement of remission using the mDAS28 cut-off of $<2.6$ (eg, baseline mDAS-follow-up mDAS). ${ }^{24} 25$ Specifically, the predictors of the ESR include tender and swollen 28 joint counts, patient's and physician's global assessments, patient's pain assessment and physical function (mHAQ). The calculation of the mDAS28 is shown as follows:

$$
\begin{aligned}
\operatorname{mDAS} 28 & =0.53 \times \sqrt{(28 \mathrm{TJC})}+0.31 \times \sqrt{(28 \mathrm{SJC})} \\
& +0.25 \times \mathrm{mHAQ}+0.001 \times \mathrm{PAIN}+0.005 \\
& \times \mathrm{EGA}+0.014 \times \mathrm{PGA}+1.694
\end{aligned}
$$

It has been previously shown that the mDAS28 performs as well as the DAS2 8 as a measure of response and disease activity. ${ }^{25}$

\section{Potential confounders}

Given concerns regarding treatment-selection bias, we used propensity score (PS) matching. A PS is the predictive probability of an outcome; in this case receiving ABA versus another anti-TNF. PS matching has been used in numerous studies to reduce selection bias in observational studies. ${ }^{26}$ In this study, the PS was estimated using a multivariable logistic regression model predicting the use of ABA versus an anti-TNF using the following key variables: age, number of prior anti-TNF agents (one prior vs two or more priors), duration of RA, swollen joint count, patient's global assessment, physician's global assessment and insurance type. We then matched on the PS (up to 1:4 match using a calliper of 0.01 ) using a greedy matching routine without replacement. ${ }^{27}$ We matched separately patients who had a follow-up at 6 months as well as those with a follow-up at 12 months. Patients could have contributed data to both groups. In addition, matching was done based on the number of available patients. Thus, for some analyses, up to four anti-TNF users were matched to one ABA user while for other analyses it was four ABA users to one anti-TNF users. Initially we performed the analyses based on prior anti-TNF use, specifically comparing PS matched ABA and anti-TNF users exposed to only one prior anti-TNF separately from PS-matched ABA and anti-TNF users exposed to two or more anti-TNFs. However, the results were not different; so we pooled the groups together.

\section{Statistical analysis}

Patient clinical and demographic characteristics were compared within the two matched cohorts (6 and 12 months). For continuous measures, means and SDs were estimated and tests were used to test statistical differences among the groups. For dichotomous measures, percentages were estimated and Fisher's 
exact test was used to test statistical differences among the groups. Response outcomes were defined based on the change in CDAI, change in mDAS28 as well as mACR20, mACR50 and mACR70 responses and meaningful change in mHAQ as defined above. Remission outcomes were based on CDAI and mDAS28. For patients who discontinued ABA or the subsequent anti-TNF agent, the last observation prior to the discontinuation was carried forward.

Comparisons of response rates were made at 6 and 12 months postinitiation using 3 -month time windows for capturing study visits. For patients with two or more visits within the window, we used the visits closest to the date of interest (6 or 12 months). Weighted mean improvement and response rates for both time points were estimated. Unadjusted and adjusted estimates comparing differences (for CDAI and mDAS28), response rates (mACR20, mACR50 and mACR70), meaningful improvement in function (mHAQ) and remission rates (CDAI and mDAS28) were performed separately using generalised linear latent and mixed models (GLLAMM). We used GLLAMM because we needed two levels of weights due to the n:1 matching and hierarchical nature of the data (eg, patients nested by physician) as these factors have been shown to influence the evaluations of treatment effectiveness. Specifically, we included multilevel weights and clustering on physician. The first level is for the patient with individual weights based on the PS matching ratio, and the second level is for the physician. We computed the second-level weights by averaging the individual probability weights within each physician. These results were reported with estimated 95\% CIs. Residual differences in baseline characteristics after matching were included in the outcome models. Patients with missing data for any of the variables in the final models were excluded from the models. Additionally, we explored potential interactions between medication selection (ABA vs anti-TNF) and number of prior anti-TNF agents in the models.
Additional sensitivity analyses were carried out, which included re-running the analyses with 1:1 matching. To assess whether responses differed based on previous experience with an anti-TNF, we re-ran the analyses (both 1:n and 1:1 matching approaches) restricting it to patients who had an inadequate clinical response to the prior anti-TNF; specifically we excluded patients who switched for safety, tolerability or other reasons from this sensitivity analysis. All analyses were conducted using STATA V.10 and 11.

\section{RESULTS}

There were 3076 RA patients who met inclusion and exclusion criteria including prior exposure to one or more anti-TNFs, initiation of either of ABA $(n=968)$ or a subsequent anti-TNF $(n=2108)$ without prior use of a non-TNF biologic (figure 1). Among those with a follow-up appointment at 6 months following initiation, there were $440 \mathrm{ABA}$ users and 958 anti-TNF users. Propensity matching was performed for those with a 6-month follow-up appointment, resulting in $431 \mathrm{ABA}$ and 746 anti-TNF users of which $66 \%$ had been exposed to only one prior anti-TNF (table 1). After matching there was better balance across most baseline patient characteristics; however, there was still imbalance across several covariates, including medicare insurance, rheumatoid factor seropositivity, disease duration, mHAQ and concomitant prednisone use, which were added as individual covariates to the outcome models. For follow-up at 12 months, there were 327 ABA users and 637 anti-TNF users. Those with follow-up at 12 months were PS matched, resulting in $311 \mathrm{ABA}$ users and 493 anti-TNF users of whom $68 \%$ had exposure to only one prior anti-TNF and there was good balance across most baseline characteristics except for disease duration that was added to as an individual covariate to the outcome models (see online supplementary table for the cohort with follow-up at 12 months). There were 272 ABA users and 531 anti-TNF users who contributed to both the 6-month and 12-month cohorts.

Figure 1 Selection of the study cohorts.

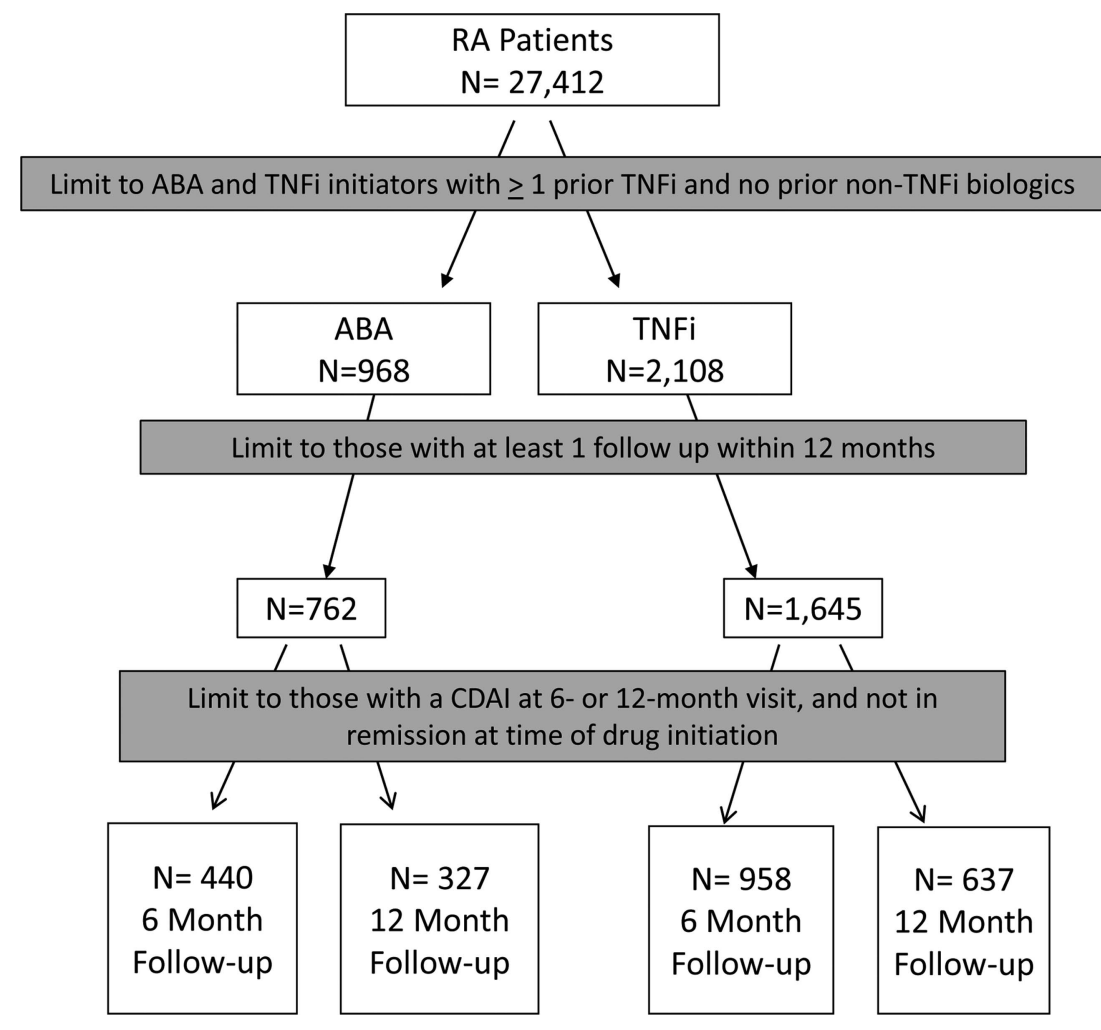


Among the ABA users, $21.5 \%$ discontinued the medication by 6 months, and by 12 months it was $27.6 \%$. In anti-TNF users, $36.6 \%$ discontinued the medication by 6 months and $38.2 \%$ by 12 months. Among those who discontinued, toxicity was reported by $5.6 \%$ of $\mathrm{ABA}$ users by 6 months and $3.0 \%$ by 12 months. For anti-TNF users, it was $13.0 \%$ by 6 months and $9.0 \%$ by 12 months.

At 6 months, response rates based on mean change in CDAI were similar in ABA users as compared with anti-TNF users (-6.1 vs -5.6$)$ in both unadjusted and adjusted analyses (figure 2). Achievement of a mACR2 20 occurred in $31.7 \%$ of ABA users and $28.2 \%$ of anti-TNF users, which was not significant in unadjusted or adjusted analyses. Achievement of a mACR50 was more likely in ABA users as compared with anti-TNF users (19.7\% vs $14.5 \%$; adjusted OR of $1.40 ; 95 \%$ CI 1.05 to 1.85$)$. The mACR70 response rates were similar, occurring in $8.6 \%$ of ABA users and $6.8 \%$ of anti-TNF users, which were not significantly different in both unadjusted and adjusted analyses. Achievement of a clinically meaningful improvement in mHAQ was similar, occurring in $33.1 \%$ of $\mathrm{ABA}$ users and $30.2 \%$ of anti-TNF users. At 12 months, response rates based on change in CDAI seemed to favour anti-TNF users with borderline significance (difference in mean change of CDAI of $-1.64 ; 95 \%$ CI -3.47 to 0.19 ). Between $35 \%$ and $37 \%$ of both groups achieved a mACR20, 20-22\% a mACR50 and 10-12\% a mACR70; none of these differences were significant in unadjusted or adjusted analyses. Achievement of a meaningful clinical improvement in function occurred similarly in the two groups $(28.9 \%$ of ABA users and $29.8 \%$ of anti-TNF users).
Remission rates based on CDAI were similar at 6 months, occurring in $9 \%$ of $\mathrm{ABA}$ users and $10 \%$ of anti-TNF users (figure 3 ). At 12 months, remission rates were $12-14 \%$ among both groups based on CDAI.

Results were similar when using the change in mDAS28 $(0.81$ vs $0.68 ; p=0.13)$ at 6 and 12 months $(1.03$ vs $1.03 ; p=0.93)$. Achievement of mDAS28 remission occurred more often in ABA users (20.2\% vs $17.3 \%)$ at 6 months, which was significant in adjusted analyses (OR 1.55 ; 95\% CI 1.01 to 2.36). To further explore whether outcomes in the two groups differed based on response to the prior anti-TNF, we re-ran the analyses in those patients who discontinued their prior anti-TNF due to lack of efficacy and similar results were found (see online supplementary figures). Additionally, we explored the robustness of our results using two strategies to ensure our matching protocol did not influence the findings. First, we redid the analyses using 1:1 matching as well as evaluating the 6-month and 12-month outcomes in a single cohort of patients who were seen both at 6 and 12 months. Again, the results were similar to those found with the 1:n matched cohort presented here.

\section{DISCUSSION}

Using data from CORRONA, a large US-based RA registry, we compared the clinical effectiveness of ABA versus a subsequent anti-TNF agent among RA patients with previous anti-TNF exposure. Specifically, we compared change in disease activity and remission rates based on CDAI and mDAS28 as well as ACR response rates and meaningful improvement in function. Both treatments were effective for the primary outcome of

Table 1 Baseline characteristics of unmatched and matched cohorts using abatacept or an anti-TNF with follow-up at 6 months

\begin{tabular}{|c|c|c|c|c|c|c|}
\hline & \multicolumn{2}{|l|}{ Unmatched } & \multirow[b]{2}{*}{$\mathrm{p}$ Value } & \multicolumn{2}{|l|}{ Matched } & \multirow[b]{2}{*}{ p Value } \\
\hline & $\begin{array}{l}A B A \\
(N=440)\end{array}$ & $\begin{array}{l}\text { Anti-TNF } \\
(\mathrm{N}=958)\end{array}$ & & $\begin{array}{l}\text { ABA } \\
(\mathrm{N}=431)\end{array}$ & $\begin{array}{l}\text { Anti-TNF } \\
(\mathrm{N}=746)\end{array}$ & \\
\hline \multicolumn{7}{|l|}{ Demographics } \\
\hline Age (mean age, SD) & $57.7(12.4)$ & $55.6(12.5)$ & 0.003 & $57.6(12.4)$ & $57.2(11.7)$ & 0.578 \\
\hline Gender (\% female) & 82.7 & 80.0 & 0.242 & 82.4 & 79.1 & 0.196 \\
\hline Race (\% white) & 81.8 & 85.4 & 0.097 & 82.4 & 85.1 & 0.216 \\
\hline \multicolumn{7}{|l|}{ Insurance (\%) } \\
\hline Private & 78.6 & 79.3 & 0.830 & 79.4 & 81.0 & 0.542 \\
\hline Medicare & 36.8 & 29.8 & 0.012 & 36.4 & 30.8 & 0.053 \\
\hline Medicaid & 5.0 & 6.9 & 0.188 & 5.1 & 6.4 & 0.374 \\
\hline \multicolumn{7}{|l|}{ Clinical } \\
\hline Rheumatoid factor seropositivity (\%) & 71.3 & 78.0 & 0.032 & 70.7 & 80.0 & 0.006 \\
\hline Disease duration (mean years, SD) & $13.4(10.3)$ & $11.5(9.4)$ & 0.000 & $13.3(10.0)$ & $12.1(9.8)$ & 0.045 \\
\hline Tender joints, N (SD) & $7.4(7.1)$ & $7.3(7.1)$ & 0.717 & $7.4(7.1)$ & $7.3(7.1)$ & 0.811 \\
\hline Swollen joints, N (SD) & $6.8(6.0)$ & $5.9(5.3)$ & 0.005 & $6.7(5.9)$ & $6.1(5.2)$ & 0.087 \\
\hline Patient pain score (mean, SD) & $52.7(25.3)$ & $49.3(25.8)$ & 0.023 & $52.7(25.5)$ & $50.2(25.8)$ & 0.112 \\
\hline mHAQ (mean, SD) & $0.7(0.5)$ & $0.6(0.5)$ & 0.012 & $0.7(0.5)$ & $0.6(0.5)$ & 0.047 \\
\hline CDAI (mean, SD) & $22.9(14.1)$ & $21.4(13.2)$ & 0.057 & $22.8(14.1)$ & $21.7(12.9)$ & 0.182 \\
\hline Disease activity* (\%) & & & 0.210 & & & 0.354 \\
\hline Low & 19.8 & 20.6 & & 20.2 & 19.0 & \\
\hline Moderate & 35.7 & 39.8 & & 35.7 & 40.0 & \\
\hline High & 44.6 & 39.7 & & 44.1 & 41.0 & \\
\hline \multicolumn{7}{|l|}{ Concomitant medications } \\
\hline Corticosteroids, (\%) & 40.0 & 34.0 & 0.036 & 39.4 & 33.0 & 0.027 \\
\hline Leflunomide, (\%) & 12.5 & 12.7 & 0.931 & 12.5 & 13.3 & 0.787 \\
\hline MTX, (\%) & 54.8 & 57.1 & 0.417 & 55.2 & 55.5 & 0.951 \\
\hline Dose, mg/week (SD) & $16.3(6.2)$ & $16.4(6.0)$ & 0.843 & $16.3(6.2)$ & $16.2(5.9)$ & 0.906 \\
\hline
\end{tabular}


Figure 2 Adjusted* response rates at 6 and 12 months among abatacept and anti-tumour necrosis factor (anti-TNF) users. *Adjusted for number of prior anti-TNF medications, baseline disease activity, rheumatoid arthritis medications. Adjusted mean difference in Clinical Disease Activity Index (CDAl) at 6 months was $0.46(95 \% \mathrm{Cl}$ -0.82 to 1.73$)$ and at 12 months $1.64(95 \% \mathrm{Cl}-3.47$ to 0.19$)$. Adjusted OR for modified American College of Rheumatology (mACR)20 response at 6 months was 1.05 (95\% Cl 0.81 to 1.36) and at 12 months $0.87(95 \% \mathrm{Cl}$ 0.59 to 1.29). Adjusted OR for mACR50 response at 6 months was $1.40(95 \% \mathrm{Cl} 1.05$ to 1.85$)$ and at 12 months 0.86 (95\% Cl 0.58 to 1.27 ). Adjusted OR for $\mathrm{mACR} 70$ response at 6 months was 1.72 (95\% Cl 0.84 to $3.53)$ and at 12 months $1.12(95 \% \mathrm{Cl}$ 0.56 to 2.24). Adjusted OR for meaningful change in modified Health Assessment Questionnaire (mHAQ) at 6 months was 1.15 (95\% Cl 0.86 to 0.48 to 1.15 ). disease severity and concomitant $1.54)$ and at 12 months $0.74(95 \% \mathrm{Cl}$

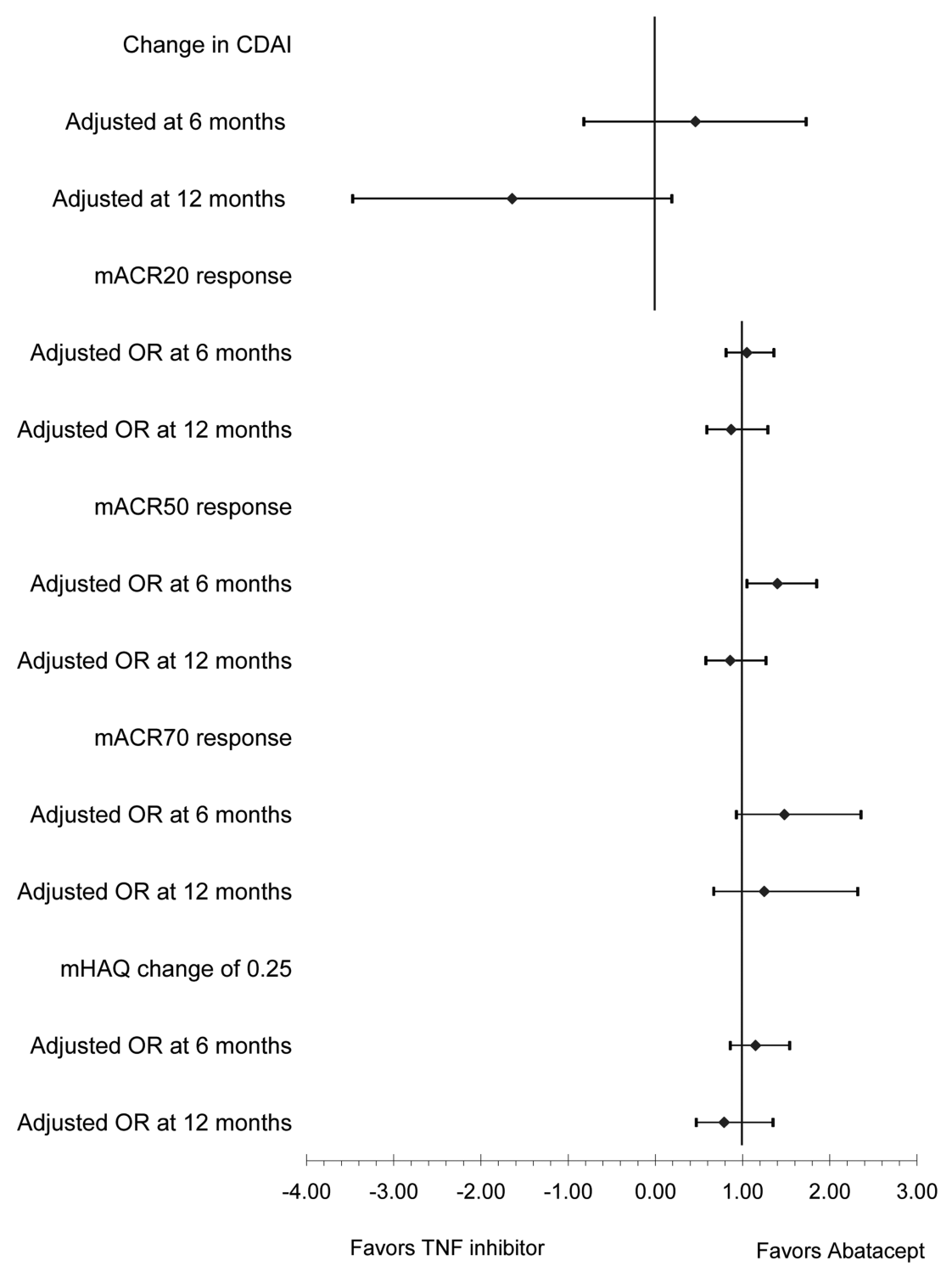

interest as the minimally clinically important difference for change in CDAI is $4.3 .^{20}$ We examined effectiveness based on several different outcome measures (change in disease activity, mACR response, meaningful improvement in mHAQ and remission) and timeframe. For the vast majority of the analyses, effectiveness was similar in the two treatment arms. This is further supported by our sensitivity analysis in which we limited the sample to those who discontinued their prior anti-TNF due to lack of efficacy and found similar results.

While our results stem from an observational cohort, they are similar to two recent systematic reviews based on clinical trials that compared the efficacy of ABA with other biologic agents using Bayesian network meta-analysis methods ${ }^{9} 28$ in inadequate responders to MTX. In the first review, ABA was compared with TNF and non-TNF biologics and found to have similar ACR50 and remission rates ${ }^{9}$ at 24 and 52 weeks. In the second review, ABA was compared with anti-TNF agents with similar responses to therapy found based on change in HAQ and ACR response rates at 6 months. ${ }^{28} \mathrm{~A}$ recently published systematic review of randomised clinical trials in patients with inadequate response to nbDMARDs found that use of ABA as compared with anti-TNFs was associated with equivalent ACR20 and ACR50 responses at 6 and 12 months, but there was a higher likelihood of achieving an ACR70 response and DAS28 remission at
12 months with ABA. $^{29}$ In contrast, a meta-analysis of randomised clinical trials of patients with inadequate response to MTX suggested a higher probability of achieving an ACR50 response with anti-TNFs as compared with ABA. ${ }^{8}$ periods for the selection of relevant studies and analysis techniques. In a recent head-to-head comparison of the addition of ABA versus adalimumab in biologic-naïve patients on background MTX, no meaningful differences in efficacy were found. ${ }^{14}$ Additionally, ABA has been compared with anti-TNF agents in the MTX-naïve early RA population with no differences in time to response and response achieved over a 1-year time period. ${ }^{30} 31$ Our findings complement these studies as according to our knowledge it is the first study that examines comparative effectiveness in those with prior exposure to anti-TNF.

Currently, it is not possible to predict which patients will respond to which particular biologic agent. However, this study enough to improve disease activity. When discussing next therapeutic interventions in those not responding or unable to take their current anti-TNF agent, these results can potentially contribute to the discussion as patient weigh other factors such potential adverse events associated with the different agents, These differences may in part be related to different time suggests that just changing the mechanism of action may not be 
CDAl remission

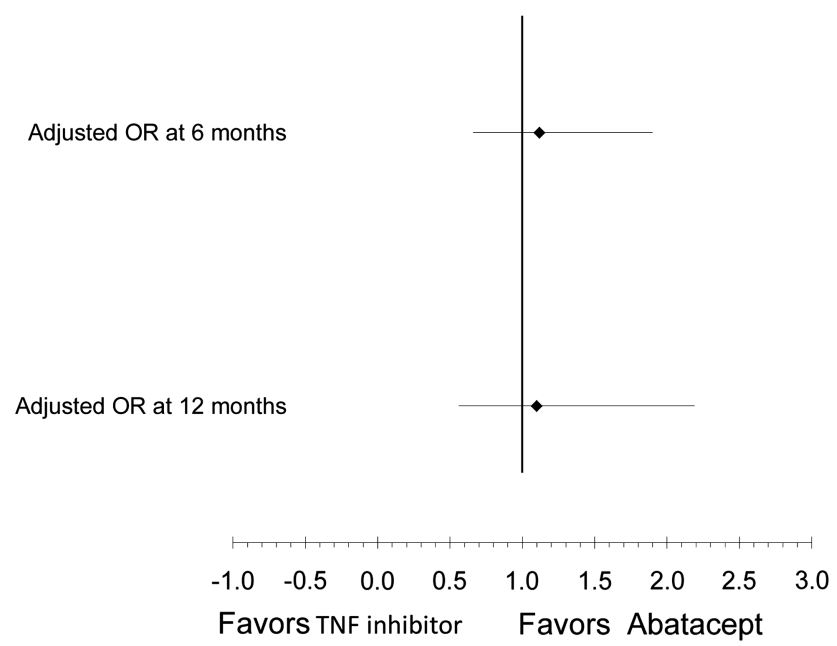

Figure 3 Adjusted* remission rates at 6 and 12 months among abatacept and anti-tumour necrosis factor users. *Adjusted for number of prior TNR, baseline disease activity, rheumatoid arthritis disease severity and concomitant medications. Adjusted mean OR for Clinical Disease Activity Index remission at 6 months was 1.21 (95\% Cl 0.76 to 1.94) and at 12 months $-1.03(95 \% \mathrm{Cl} 0.61$ to 1.75$)$.

out-of pocket costs and medication route of administration that impacts convenience. The current availability of ABA as an intravenous infusion and subcutaneous injection does allow more flexibility for the patient's preference.

Our study has several strengths. We utilised a national US-based registry of RA patients with physician-derived outcome measures to examine comparative effectiveness in patients with prior anti-TNF exposure. We examined drug response (based on change in disease activity, achievement of a modified ACR response and achievement of a meaningful improvement in mHAQ) and remission. In addition, we used both CDAI and mDAS28 to assess response and remission. This work focused on the real-world' effectiveness of agents in US patients, who are markedly different than RCT subjects in terms of comorbidities and RA disease activity, and more representative of patients found in clinical practice. ${ }^{32}$ Finally, we were able to use advanced epidemiologic methods (eg, PSs) to compare responses to the different agents when selection bias exists.

However, there are limitations to our study. The timing of the study visits was based upon clinic visits and not mandated as occurs in RCTs that influenced which patients were included based on follow-up visits. Of note, the mean study interval between study visits was approximately 4.5 months. Most of the patients were white, which limits generalisability to other racial groups. In addition, acute phase reactant data were not available for all patients in the study. As a result, we applied previously validated outcome measures not requiring acute phase reactants such as CDAI and mDAS for response and remission outcomes as well as modified ACR response rates and mHAQ scores. ${ }^{20} 2225$ While PS matching improved the balance between the cohorts to most measure covariates, it is possible that there is still residual confounding for unmeasured covariates.

In summary, the results of this US-based observational study indicate similar rates of drug response and remission with use of $\mathrm{ABA}$ and anti-TNF agents among those with prior anti-TNF exposure. These findings were unchanged when we limited the analyses to those who failed their prior anti-TNF due to lack of efficacy. While we saw no difference when comparing ABA with anti-TNF agents, other non-TNF biologics with different mechanisms of action may be associated with superior outcomes as compared with anti-TNFs. Further comparative effectiveness studies are necessary to evaluate the benefits of switching to a different non-TNF biologic versus anti-TNF intraclass switching among patients with an inadequate response to an anti-TNF.

Contributors All authors were responsible for the study design and revision and approval of the final manuscript.JMK and JDG were responsible for the acquisition of data.LRH, GWR and JDG were responsible for the analysis of data. LRH was responsible for drafting of manuscript.

Funding In the past 2 years, the Consortium of Rheumatology Researchers of North America (CORRONA) has received support from Abbott, Amgen, Astra-Zeneca, Genentech, Janssen (Centocor), Eli Lilly and Pfizer through contracted subscriptions to the database.

Competing interests LRH: CORRONA. GWR: CORRONA. JMK: CORRONA, AbbVie, Amgen, BMS, Genentech, Lilly, Pfizer, UCB. JRC: Roche, Genentech, UCB, Janssen, CORRONA, Amgen, Pfizer, BMS, Crescendo, AbbVie. DHS: Lilly, CORRONA, Pfizer, Amgen, UpToDate. MCH: AbbVie, Amgen, Bristol Myers Squibb, Genentech/ Roche. JDG: CORRONA, Astra Zeneca, Novartis, Pfizer. The study design, data analysis and reporting of results in this manuscript were performed independent of all funding sources. Some of the investigators received support from the National Institutes of Health (LRH AR053856; JRC AR053351; JDG AR054412; DHS AR055989 and AR047782). In addition, DHS has research grants from AHRQ, the Arthritis Foundation, Amgen and Lilly. JRC has research grants from AHRQ (R01 HS018517). JDG has research funding from Agency for Healthcare Research and Quality and the Arthritis National Research Foundation.

Ethics approval Local Institutional Review Boards.

Provenance and peer review Not commissioned; externally peer reviewed.

Open Access This is an Open Access article distributed in accordance with the Creative Commons Attribution Non Commercial (CC BY-NC 3.0) license, which permits others to distribute, remix, adapt, build upon this work non-commercially, and license their derivative works on different terms, provided the original work is properly cited and the use is non-commercial. See: http://creativecommons.org/ licenses/by-nc/3.0/

\section{REFERENCES}

1 Helmick CG, Felson DT, Lawrence RC, et al. Estimates of the prevalence of arthritis and other rheumatic conditions in the United States. Part I. Arthritis Rheum 2008; $58: 15-25$

2 Smolen JS, Aletaha D, Machold KP. Therapeutic strategies in early rheumatoid arthritis. Best Pract Res Clin Rheumatol 2005;19:163-77.

3 van Vollenhoven RF, Ernestam S, Geborek P, et al. Addition of infliximab compared with addition of sulfasalazine and hydroxychloroquine to methotrexate in patients with early rheumatoid arthritis (Swefot trial): 1-year results of a randomised trial. Lancet 2009:374:459-66.

4 Finckh A, Ciurea A, Brulhart $L$, et al. B cell depletion may be more effective than switching to an alternative anti-tumor necrosis factor agent in rheumatoid arthritis patients with inadequate response to anti-tumor necrosis factor agents. Arthritis Rheum 2007;56:1417-23.

5 Gomez-Reino JJ, Maneiro JR, Ruiz J, et al. Comparative effectiveness of switching to alternative tumour necrosis factor (TNF) antagonists versus switching to rituximab in patients with rheumatoid arthritis who failed previous TNF antagonists: the MIRAR Study. Ann Rheum Dis 2012;71:1861-4.

6 Kekow J, Mueller-Ladner U, Schulze-Koops H. Rituximab is more effective than second anti-TNF therapy in rheumatoid arthritis patients and previous TNFalpha blocker failure. Biologics: Targets Ther 2012;6:191-9.

7 Blom M, Kievit W, Donders AR, et al. Effectiveness of a third tumor necrosis factor-alpha-blocking agent compared with rituximab after failure of 2 TNF-blocking agents in rheumatoid arthritis. J Rheumatol 2011;38:2355-61.

8 Salliot C, Finckh A, Katchamart W, et al. Indirect comparisons of the efficacy of biological antirheumatic agents in rheumatoid arthritis in patients with an inadequate response to conventional disease-modifying antirheumatic drugs or to an anti-tumour necrosis factor agent: a meta-analysis. Ann Rheum Dis 2011;70:266-71.

9 Guyot P, Taylor P, Christensen R, et al. Abatacept with methotrexate versus other biologic agents in treatment of patients with active rheumatoid arthritis despite methotrexate: a network meta-analysis. Arthritis Res Ther 2011;13:R204

10 Guyot P, Taylor PC, Christensen R, et al. Indirect treatment comparison of abatacept with methotrexate versus other biologic agents for active rheumatoid arthritis despite methotrexate therapy in the United kingdom. J Rheumatol 2012;39:1198-206. 
11 Schiff M, Keiserman M, Codding C, et al. Efficacy and safety of abatacept or infliximab vs placebo in ATTEST: a phase III, multi-centre, randomised, double-blind, placebo-controlled study in patients with rheumatoid arthritis and an inadequate response to methotrexate. Ann Rheum Dis 2008;67:1096-103.

12 Kremer JM, Westhovens $\mathrm{R}$, Leon $\mathrm{M}$, et al. Treatment of rheumatoid arthritis by selective inhibition of T-cell activation with fusion protein CTLA4lg. N Engl J Med 2003;349:1907-15.

13 Kremer JM, Genant HK, Moreland LW, et al. Effects of abatacept in patients with methotrexate-resistant active rheumatoid arthritis: a randomized trial. Ann Intern Med 2006;144:865-76.

14 Schiff M, Weinblatt M, Valente R, et al. Head-to-head comparison of subcutaneous abatacept versus adalimumab for rheumatoid arthritis: two-year efficacy and safety findings from AMPLE trial. Ann Rheum Dis 2014;73:86-94.

15 Siegel J. Comparative effectiveness of treatments for rheumatoid arthritis. Ann Intern Med 2008;148:162-3.

16 Greenberg JD, Bingham CO 3rd, Abramson SB, et al. Effect of cardiovascular comorbidities and concomitant aspirin use on selection of cyclooxygenase inhibitor among rheumatologists. Arthritis Rheum 2005;53:12-17.

17 Kremer J. The CORRONA database. Ann Rheum Dis 2005;64(Suppl 4):iv37-41.

18 Felson DT, Anderson JJ, Boers $M$, et al. The American College of Rheumatology preliminary core set of disease activity measures for rheumatoid arthritis clinical trials. The Committee on Outcome Measures in Rheumatoid Arthritis Clinical Trials. Arthritis Rheum 1993;36:729-40.

19 Aletaha D, Nell VPK, Stamm T, et al. Acute phase reactants add little to composite disease activity indices for rheumatoid arthritis: validation of a clinical activity score. Arthritis Res Ther 2005;7:R796-806.

20 Greenberg JD, Harrold LR, Bentley MJ, et al. Evaluation of composite measures of treatment response without acute-phase reactants in patients with rheumatoid arthritis. Rheumatology (Oxford) 2009;48:686-90.

21 Saag KG, Teng GG, Patkar NM, et al. American College of Rheumatology 2008 recommendations for the use of nonbiologic and biologic disease-modifying antirheumatic drugs in rheumatoid arthritis. Arthritis Rheum 2008:59:762-84.

22 Goldman JA, Xia HA, White B, et al. Evaluation of a modified ACR20 scoring system in patients with rheumatoid arthritis receiving treatment with etanercept. Ann Rheum Dis 2006;65:1649-52.
23 Maska L, Anderson J, Michaud K. Measures of functional status and quality of life in rheumatoid arthritis: Health Assessment Questionnaire Disability Index (HAQ), Modified Health Assessment Questionnaire (MHAQ), Multidimensional Health Assessment Questionnaire (MDHAQ), Health Assessment Questionnaire II (HAQ-II), Improved Health Assessment Questionnaire (Improved HAQ), and Rheumatoid Arthritis Quality of Life (RAQoL). Arthritis Care Res 2011;63(S11): S4-13.

24 Prevoo ML, van 't Hof MA, Kuper $\mathrm{HH}$, et al. Modified disease activity scores that include twenty-eight-joint counts. Development and validation in a prospective longitudinal study of patients with rheumatoid arthritis. Arthritis Rheum 1995:38:44-8.

25 Bentley MJ, Greenberg JD, Reed GW. A modified rheumatoid arthritis disease activity score without acute-phase reactants (mDAS28) for epidemiological research. J Rheumatol 2010;37:1607-14.

26 Austin PC. A critical appraisal of propensity-score matching in the medical literature between 1996 and 2003. Stat Med 2008;27:2037-49.

27 Rassen JA, Shelat AA, Myers J, et al. One-to-many propensity score matching in cohort studies. Pharmacoepidemiol Drug Saf 2012;21(Suppl 2):69-80.

28 Guyot P, Taylor PC, Christensen RP, et al. Indirect Treatment Comparison of Abatacept with Methotrexate Versus Other Biologic Agents for Active Rheumatoid Arthritis Despite Methotrexate Therapy in the United Kingdom. J Rheumatol 2012:39:1198-206.

29 Hochberg MC, Berry S, Broglio K, et al. Mixed treatment comparison of efficacy and tolerability of biologic agents in patients with rheumatoid arthritis. Curr Med Res Opin 2013;29:1213-22

30 Yazici Y, Filopoulos MT, Swearingen CJ. Comparative Effectiveness and Time to Response Among Abatacept, Adalimumab, Etanercept and Infliximab for the Treatment of Rheumatoid Arthritis in a Real World Routine Care Registry. [abstract]. Arthritis Rheum 2011;63(Suppl 10):2233

31 Yazici Y, Swearingen C, Nadkarni A, et al. Comparative Efficacy and Tolerability of Biologic Therapies in Early Rheumatoid Arthritis Utilizing a Bayesian Approach. [abstract]. Arthritis Rheum 2011;63(Suppl 10):1240.

32 Greenberg JD, Kishimoto M, Strand V, et al. Tumor necrosis factor antagonist responsiveness in a United States rheumatoid arthritis cohort. Am J Med 2008;121:532-8. 